\title{
Shifting human color memory
}

\author{
ELIZABETH F. LOFTUS \\ University of Washington, Seattle, Washington 98195
}

\begin{abstract}
Persons who witnessed an automobile accident involving a green car were exposed to information that the car was blue. On a subsequent color recognition test, most subjects shifted their color selection in the direction of the misleading information and away from the actual perceived color. Shifting was greater for subjects who did not initially commit themselves to a color selection. Control subjects, who were not exposed to misleading information, distributed their choices around the true color, and did not show a systematic color shift over time.
\end{abstract}

When people witness important events, such as tratfic accidents, they are occasionally exposed to subsequent information that can influence the memory of that event. This occurs even when the initial event is largely visual, and the additional information is presented in a verbal format (Loftus, 1975; Pezdek, 1977). To illustrate, college students were presented with films of complex fast-moving events and immediately afterward were asked a series of questions. Some of the questions were designed to present misleading information, that is, to suggest the existence of an object that did not exist. Thus, the students might be asked, "How fast was the white sports car going when it passed the barn while traveling along the country road?" when no barn existed. A control question asked other students, "How fast was the white sports car going while traveling along the country road?" All students were subsequently asked whether they had seen the presupposed object; it was found that the misleading questions increased the likelihood that the students would later report having seen a nonexistent object. The questions are apparently effective because they contain information (in this case, false information) which is integrated into the memorial representation of the event, thereby supplementing or altering that representation.

Mentioning an object during the course of an interrogation causes some people to later report having seen that object. For example, $17 \%$ of those asked the "barn" question later reported they had seen a barn; $83 \%$ still claimed they had not. Does this mean that these sorts of manipulations affect only a small percentage of people? Not necessarily. The design of the previous experiments only permitted the subjects to make a binary response; they could say "yes" or "no." In the present experiment, a recognition procedure was used which allowed the subjects to make any one of a large number of responses. This

This research was supported by a grant from the National Institute of Mental Health. Requests for reprints should be sent to Elizabeth Loftus, Department of Psychology, University of Washington, Seattle, Washington 98195. procedure allowed more sensitive observation of the extent to which a group of individuals, and even a single individual, is influenced by misleading information to which he or she has been exposed.

\section{EXPERIMENT 1}

\section{Method}

Subjects. One hundred undergraduates at the University of Washington participated for course credit. They were run in groups ranging from two to eight in size. Only subjects who reported no color blindness were used in this study.

Design and procedure. A series of 30 color slides, depicting successive stages in an auto-pedestrian accident, was shown for $3 \mathrm{sec}$ each. A red Datsun was seen traveling along a side street toward an intersection. It then turned right and knocked down a pedestrian who was crossing at the crosswalk. A green car drove past the accident, but did not stop. The green car appeared in only a single slide (see Figure 1). Immediately after viewing the slides, the subjects answered a series of 12 questions. For half of the subjects, Question 10 was, "Did the blue car that drove past the accident have a ski rack on the roof?" The remaining subjects were asked the same question with the word "blue" deleted. All subjects participated in a 20 -min filler activity, which required them to read an unrelated story and answer some questions about it. Finally, a color recognition test was administered. The subjects were shown a color wheel containing 30 color strips, each of which was numbered. Numbers 1-5 corresponded to shades of purple, 6-10 to blue, 11-15 to green, 16-20 to yellow, 21-25 to orange, and 26-30 to red. These corresponded to wavelengths in the visible range from approximately $425 \mathrm{~nm}$ to $675 \mathrm{~nm}$. Nearly equal increments of wavelengths separated the various strips. The subjects were given a list of 10 objects and for each object their task was to pick the color that best represented their recollection of the object and to write down the color number next to the name of each object. The critical item, the green car that passed the accident, was closest to the color strip numbered 13. This strip corresponded to a wavelength of approximately $520 \mathrm{~nm}$.

In order to determine whether a single individual who is exposed to misleading information will shift his or her color estimate in the direction of that misleading information, the control group was asked to return 7 days later. Without reviewing the slides, they completed another questionnaire. Forty-six subjects returned. Unbeknownst to them, they were divided into two groups which were matched for their initial color choice. The new 12-item questionnaire was identical to the original one for one group, and for the other group the key question was changed to read, "Did the blue car that drove past the accident have a ski rack on the roof?" In other words, one group remained a control group and the other was now 


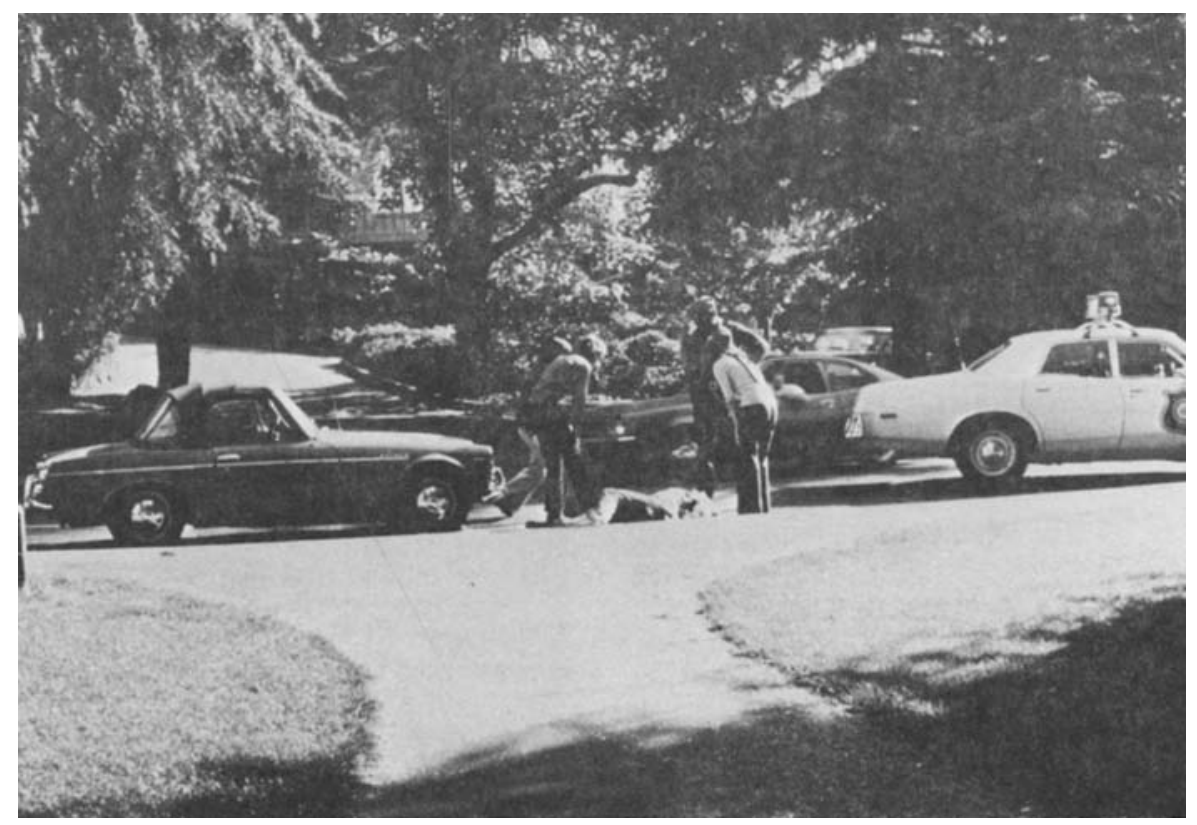

Figure 1. Black-and-white copy of 1 of 30 color slides shown to experimental subjects. The critical item was a green car seen at the center of this slide.

"told" that the car was blue. A new color recognition test followed.

\section{Results}

Immediate color memory. First, the immediate color choices for those subjects presented with the misleading "blue" information and those who were not (controls) were compared. These data are shown in Figure 2. Relative to the controls, the "blue" subjects selected "bluer" colors. Of the 50 control subjects, 2 selected a yellow-green, 37 selected a green (11-15), 8 selected a blue (6-10), and 3 selected some totally unrelated color (e.g., red). The "blue" subjects responded differently: Twenty-four selected a green color (with the majority selecting a bluish green), 24 selected a blue color, and 2 selected a totally unrelated color. These two subjects, as well as the three from the control group, were omitted from further data analysis. The mean selection of 47 subjects from the control was 12.55 , whereas the 48 "blue" subjects produced a mean of 10.19 (Mann-Whitney $U$ test yielded $z=4.7, p<.01$ ). Thus, the introduction of the false color information significantly affected the ability of a group of subjects to correctly identify a color they had seen before.

As Figure 2 indicates, $28 \%$ of the subjects in the control group (or 13 out of 47 ) showed perfect color memory in that they responded by selecting Color Strip 13 (assuming no bias toward that strip). This indicates an unusual degree of accuracy for what might appear to be an incidental detail. In the "blue" group, only $8 \%$ of the subjects (or 4 out of 48 ) showed perfect color memory. This implies that the misleading information shifted over two-thirds (a lower bound estimate) of those subjects who had demonstrated perfect memory. Thus, the data show that not only can a sizable proportion of the subjects be shifted, but the subjects who are shifted are not limited to those with fuzzy memories.

Delayed color memory. Recall that the control subjects were matched according to their initial color choice and subsequently either exposed to "blue" information or no additional color information. Each subject's second color choice was then compared with

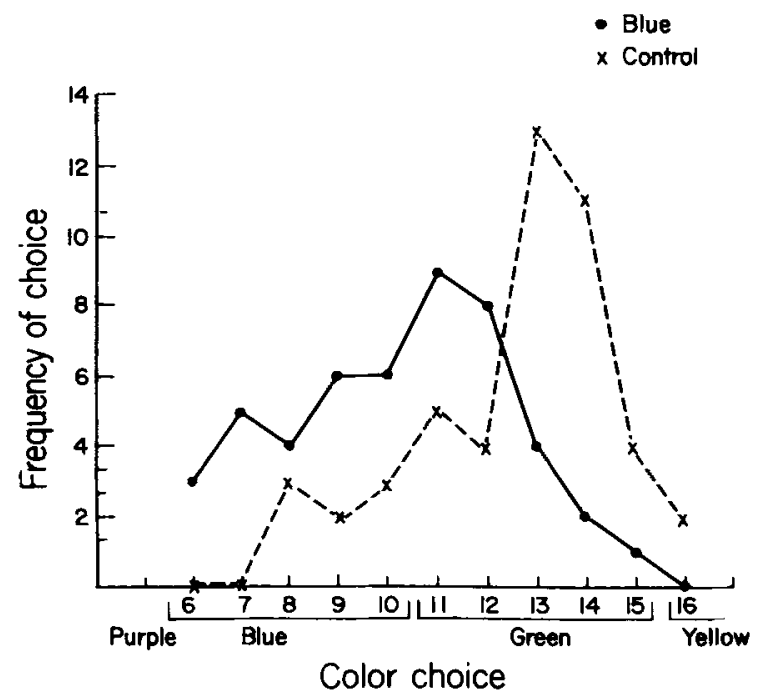

Figure 2. Frequency of color choices for subjects who were exposed to misleading information ("blue") and subjects who were not (control). 
his or her initial choice. In addition to showing the number of subjects whose two choices were the same, Figure 3 indicates the number of subjects shifting toward blue, the number shifting away from blue, and the degree of these shifts. Again, the "control-blue" subjects shifted toward "bluer" colors, relative to the control-control subjects. In the latter group, there were four ties, 9 shifts toward blue, and 10 shifts away from blue. For the control-blue subjects, there were five ties, 14 shifts in the direction of blue, and 4 shifts away from blue. There was not a significant shift toward blue in the control-control group, whereas there was in the controlblue group (by sign test). The mean selection of 23 subjects from the control-control group was 12.86 , whereas it was 11.04 for the 23 control-blue subjects. Mann-Whitney $U$ tests indicated that, while the control. blue subjects shifted significantly more than the controlcontrols $(p<.01)$, their selections were somewhat less blue than the original blue subjects (11.04 vs. 10.19 , $.10<\mathrm{p}<.05)$. This probably indicates the greater difficulty involved in shifting a person who has already made an overt selection.

The conclusions drawn here do not change if the analysis is limited to those subjects who initially responded by selecting a color strip in the green range. Eighteen control-controls and 19 control-blues initially did so. In the former group there were four ties, five shifts toward blue, and nine shifts away from blue. In the latter group there were three ties, 13 shifts toward blue, and 3 shifts away from blue.

\section{EXPERIMENT 2}

This experiment was designed to test the hypothesis that a person who has made a color commitment will

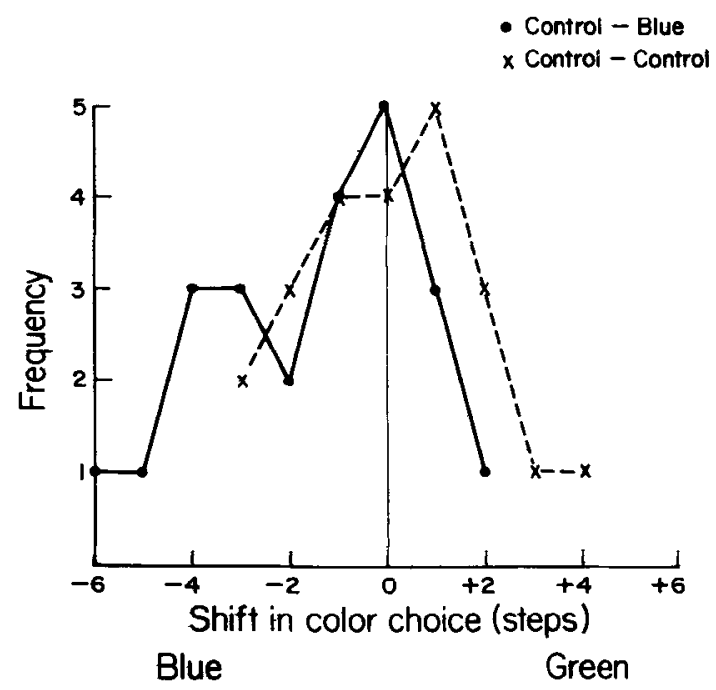

Figure 3. Frequency with which subjects shifted either toward blue or away from it, between an initial color choice and a subsequent choice. Half the subjects were exposed to misleading information between their first and second choices (control-blue) and half were not (control-control).
Table 1

Mean Color Selection for Each Experimental Condition

\begin{tabular}{|c|c|c|}
\hline \multirow[b]{2}{*}{ Conditions } & \multicolumn{2}{|c|}{ Color Selection } \\
\hline & $\begin{array}{l}\text { Immedi- } \\
\text { ately }\end{array}$ & 1 Week \\
\hline & \multicolumn{2}{|c|}{ Experiment 1} \\
\hline Blue & \multicolumn{2}{|l|}{10.19} \\
\hline Control & \multicolumn{2}{|l|}{12.55} \\
\hline Control-Control & & 12.86 \\
\hline Control-Blue (with initial color test) & & 11.04 \\
\hline & \multicolumn{2}{|c|}{ Experiment 2} \\
\hline Control-Blue (with initial color test) & & 11.11 \\
\hline Control-Blue (without initial color test) & & 9.79 \\
\hline
\end{tabular}

Note-True color = 13; "bluer" response indicated by lower num. bers. Only responses in the yellow/green/blue range are included.

be more difficult to influence later on. Misleading information was hypothesized to have less of an impact.

\section{Method}

Fifty-five subjects, who reported no experience with color blindness, were shown the slide sequence and given the control questionnaire. Of these, 25 were given a color recognition test and 30 were not. Seven days later they were asked to return, given the questionnaire containing the critical "blue" question, and, finally, given a color recognition test. Of those 25 who had been given a color recognition test, 4 tailed to return and the remaining 21 were treated exactly as the control-blue subjects in Experiment 1. Of those 30 who had been given a color recognition test, 2 failed to return and the remaining 28 were treated nearly the same as the control-blue subjects, with the exception that they had not been given an initial color recognition test. These conditions are outlined in Table 1.

\section{Results}

Of the 21 subjects who had been given an initial color test, 19 chose colors in the blue-green range (6-14) and 2 chose totally unrelated colors. The mean selection for the 19 subjects whose responses were in the blue-togreen range was 11.11 . Of those 28 subjects who had not been given an initial color test, 24 chose colors in the blue-green range and 4 chose totally unrelated colors. The mean selection for the 24 subjects whose responses were in the blue-to-green range was 9.79 . The groups were different by a Mann-Whitney $U$ test $(z=1.8$, $p<.05$, one-tailed test). Thus, those not given an initial color test selected a color that was significantly bluer than those given an initial color test, indicating the greater impact of the misleading information under conditions where the subject had not already made a commitment to a particular response color.

\section{GENERAL DISCUSSION}

The mean color selections for each of the experimental conditions are shown in Table 1. To reiterate, subjects who were exposed to misleading information shifted their color selection in the direction of the misleading information and away from the actual object color. The shift appeared when comparing group averages, and also when examining individual subject 
responses. Further, a sizable percentage of the subjects actually shifted. The misleading information had a larger impact when the initial color memory had faded somewhat, and when the subject had not made a prior color selection.

To understand these results, one must accept the fact that a person's memory for some experience consists of a good deal more than the environmental input (objectively present physical stimuli) that gave rise to it. The memory is based partly on prior knowledge, and partly on information acquired subsequent to the initial experience. Information from these sources, a blend, as it were, of fact and fantasy, becomes integrated to form a single memorial experience.

It is interesting to speculate about why a subject who has already made a color response does not shift as easily as one who has not. Perhaps subjects integrate into memory their earlier responses, as well as the information provided by the questions themselves. This preliminary notion is in need of further research.

The integration process can be seen at work in other experimental examples involving color memories. Subjects looked at a lemon and a tomato both cut out of an orange piece of paper, and had to select colors from a color wheel that matched these objects. There was a marked tendency to choose a color that was more like the usual color of the cut-out object than the orange color of the paper (Branca, 1964). Lemons were seen as more yellow and tomatoes were seen as more red. In other words, the distortions were in the direction of the natural, remembered color of the objects. Similarly, subjects reported that a green felt leaf in hidden red illumination was "greener" than an identically colored felt donkey in the same illumination (Duncker, 1939). In these instances, a subject's "memory" was determined by an integration of environmental input with prior knowledge. This also occurred in a study in which playing cards with suit reversed (e.g., a red four of clubs) were presented to subjects for brief intervals of a few milliseconds (Bruner \& Postman, 1949). In many cases subjects reported seeing a red four of hearts or a black four of clubs. But occasionally, they "saw" a purple card. In another study (Thomas \& DeCapito, 1966), subjects were shown a colored test patch of light and asked to name it. For example, a blue-green ( $490 \mathrm{~nm}$ in wavelength) was shown to subjects who might name it blue or green. A recognition test was subsequently administered and it was found that those subjects who labeled the patch green remembered it as being "greener"; those who labeled it blue recalled it as "bluer." In this instance, a subject's memory was determined by an integration of environmental input with subsequent information provided by the subject.

The present work extends the earlier observations to include the influence of information acquired subsequent to the perception of a to-be-remembered detail. In the present experiments, the subsequent information was introduced in a naturalistic fashion, during the course of an interrogation of a subjectwitness. Most subjects easily integrated the misleading information and their recollections, and, more importantly, their reports of recollections, were thereby altered.

\section{REFERENCES}

Branca, A. Psychology-Science of behavior. Boston: Allyn \& Bacon, 1964.

Bruner, J. S., \& Postman, L. On the perception of incongruity: A paradigm. Journal of Personality, 1949, 18. 206-223.

DUNCKER, K. The influence of past experience upon perceptual properties. American Journal of Psychology, 1939, 52. 255-265.

LofTus. E. F. Leading questions and the eyewitness report. Cognitive Psychology, 1975, 7. 560-572.

PezdeK, K. Cross-modality semantic integration of sentence and picture memory. Journal of Experimental Psychology: Human Learning and Memory, 1977, 3. 515-524.

Thomas, E. R., \& DeCapito, A. Role of stimulus labeling in stimulus generalization. Journal of Experimental Psychology, $1966,71,913-915$.

(Received for publication June 27, 1977; revision accepted August 17, 1977.) 